\title{
Rivaroxaban e malattia renale cronica: evidenze dal presente e prospettive future
}

Giornale di Tecniche Nefrologiche e Dialitiche 2019, Vol. 3I (I) 30-36

(C) The Author(s) 2019

Article reuse guidelines:

sagepub.com/journals-permissions

DOI: $10.1177 / 0394936219836652$

journals.sagepub.com/home/gtn

(A)AGE

\author{
Luca Di Lullo', Claudio Ronco², Fulvio Floccari ${ }^{3}$, \\ Antonio De Pascalis ${ }^{4}$, Vincenzo Barbera', Rodolfo Rivera ${ }^{5}$ \\ e Antonio Bellasi ${ }^{6}$
}

\begin{abstract}
Rivaroxaban and Chronic Kidney Disease: evidence from the present and future prospects

Cardiovascular diseases represent the main cause of comorbidly in chronic kidney disease (CKD) patients, with a 7\% incidence in atrial fibrillation (AF) in end-stage renal disease (ESRD) patients.

Until recently, prophylactic treatment of atrial fibrillation complications (such as thromboembolism) was mainly based on vitamin $\mathrm{K}$ antagonists (VKA) or heparin.

In the last years, direct oral anticoagulants (DOACs) have been made available; however, their renal clearance limits their use on patients with severe renal impairment (eGFR $<15 \mathrm{~mL} / \mathrm{min}$ ). Among DOACs, Rivaroxaban, a factor $X$-activated $(\mathrm{Xa})$ inhibitor, shows good renal profile and it can be used in CKD patients up to stage 4 as well as in ESRD patients (at the moment only in North America and Canada).
\end{abstract}

\section{Keywords}

Atrial fibrillation, Chronic kidney disease, Warfarin, Direct oral anticoagulants, Rivaroxaban

\section{Introduzione}

La fibrillazione atriale (FA) rappresenta l'aritmia più frequentemente riscontrabile nella pratica clinica, con una prevalenza che aumenta progressivamente al crescere dell'età. ${ }^{1}$ Studi epidemiologici recenti suggeriscono come, nonostante le correzioni per età e altre condizioni comorbide, tale prevalenza si confermi in crescita, permettendo così di ipotizzare l'influenza di altri fattori predisponenti. ${ }^{2-6} \mathrm{La}$ malattia renale cronica (chronic kidney disease, CKD) e altre patologie cardiovascolari condividono numerosi fattori di rischio, tra i quali età, ipertensione arteriosa, e diabete, che rendono l'aritmia così diffusa nella popolazione anziana. ${ }^{7,8}$ Nell'ultimo ventennio un tale aumento nella prevalenza di FA è stata anche segnalato nei pazienti sottoposti a trattamento emodialitico. ${ }^{9}$

\section{CKD, FA e outcome cardiovascolari}

La stretta correlazione tra FA e cardiopatie rende difficile valutarne l'impatto sulla morbidità e mortalità. In confronto ai pazienti con eGFR $>59 \mathrm{~mL} / \mathrm{min}$, quelli con eGFR compreso tra 45 e $59 \mathrm{~mL} / \mathrm{min}$ hanno un rischio di morte corretto $1-2$ volte maggiore, che diventa però $5-9$ volte più elevato quando l'eGFR si riduce al di sotto dei $15 \mathrm{~mL} / \mathrm{min}^{10}$ Con riferimento alla sola condizione di FA, i risultati di studi trasversali ricavati dall'United States Renal Data System (USRDS) dimostrano che i pazienti con malattia renale allo stato terminale (end-stage renal disease, ESRD)

\footnotetext{
'UOC Nefrologia e Dialisi, Ospedale L. Parodi - Delfino, Colleferro, Italy

2International Renal Research Institute, Ospedale S. Bortolo, Vicenza, Italy

3UOC Nefrologia e Dialisi, Ospedale "S. Paolo", Civitavecchia, Italy

"UOC Nefrologia e Dialisi, Ospedale "V. Fazzi”, Lecce, Italy

${ }^{5}$ Clinica Nefrologica, Ospedale "S.Gerardo", Monza, Italy

"Department of Research, Innovation and Brand Reputation, ASST (Azienda Socio Sanitaria Territoriale) Papa Giovanni XXIII, Bergamo, Italy

Corrispondenza:

Luca Di Lullo, UOC Nefrologia e Dialisi, Ospedale "L. Parodi -

Delfino”, Piazza Aldo Moro, I, 00034 Colleferro, Roma, Italy.

E-mail: dilulloluca69@gmail.com
} 


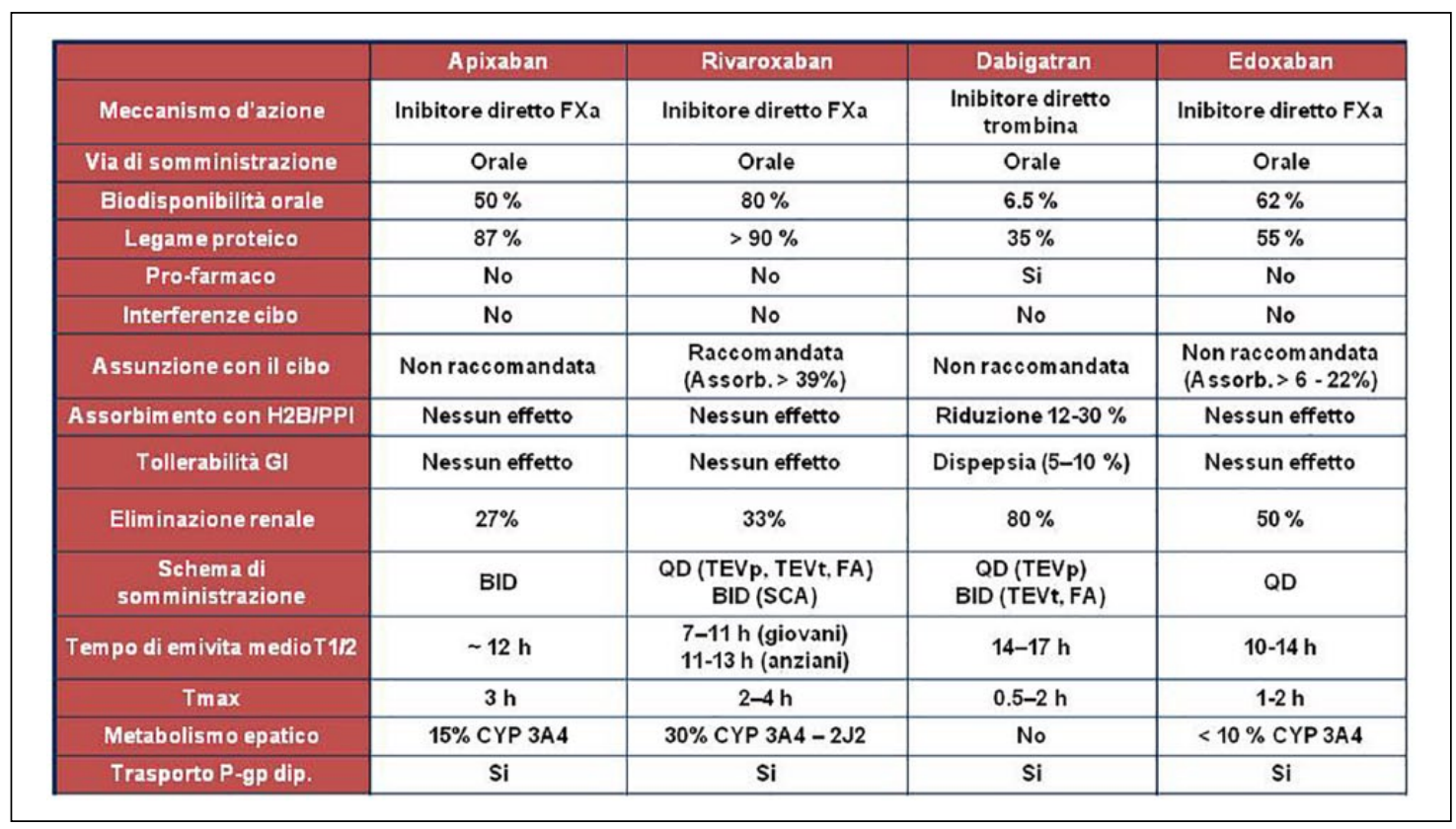

Figura I. Profilo farmacologico dei DOACs.

e FA nota hanno un tasso annuo di mortalità del $5 \%$ contro il $2 \%$ di quelli senza aritmia. ${ }^{10}$ La principale complicanza della FA è rappresentata dallo stroke ischemico. Nei diversi stadi di malattia renale cronica questo rischio diviene progressivamente più elevato. Tale dato è stato confermato anche da uno studio osservazionale giapponese (del 1977) condotto su individui sani non affetti da ESRD. In tale studio si mostrava che il rapporto di rischio per stroke nel sottogruppo con eGFR compreso tra 40 e $70 \mathrm{~mL} / \mathrm{min}$ è risultato pari a 1.9, raggiungendo il valore di 3.1 in quello con eGFR di $40 \mathrm{~mL} / \mathrm{min}$ rispetto ai soggetti con eGFR di $70 \mathrm{~mL} / \mathrm{min} .{ }^{11} \mathrm{Nel}$ trial VALIANT, comprendente pazienti con infarto acuto del miocardio e segni di insufficienza cardiaca (ma non ESRD) si è osservato un progressivo incremento dal $2 \%$ al $6 \%$ del rischio di stroke dopo 3 anni con il ridursi dell'eGFR da 75 a $45 \mathrm{~mL} / \mathrm{min} .{ }^{10}$ Nell'USRDS l'incidenza di stroke nei pazienti in emodialisi è di circa il $15.1 \%$ vs il $9.6 \%$ in quelli affetti da CKD non in emodialisi, e il $2.6 \%$ nei pazienti senza CKD. ${ }^{12}$

Relativamente alla sola FA, un'analisi monocentrica in pazienti emodializzati ha fatto rilevare un tasso di eventi tromboembolici del $24 \%$ all'anno rispetto al $5 \%$ di coloro i quali presentavano un ritmo sinusale; questi valori indicano un aumento del rischio relativo di circa 4.6 volte. ${ }^{13}$ Nell'USRDS, i soggetti con ESRD e FA avevano un rischio di stroke di 1.6 maggiore rispetto a quelli senza FA. Tale dato era riferito esclusivamente al più elevato rischio (1.8 volte) di stroke ischemico, mentre quello di stroke emorragico era simile. La modalità di trattamento sostitutivo (emodialisi o dialisi peritoneale) non sembra avere influenza sul rischio di stroke. ${ }^{12}$

\section{Anticoagulanti orali diretti e malattia renale cronica}

Le attuali evidenze scientifiche dimostrano che nei pazienti con FA e funzione renale normale la terapia anticoagulante rappresenta la più efficace forma di profilassi per l'aumentato rischio tromboembolico e/o per l'insorgenza di stroke. ${ }^{4}$ Per contro, nei pazienti con CKD, in particolar modo quelli sottoposti a terapia dialitica, l'aumento del rischio emorragico, nonché la mancanza di prove sicure a favore di un efficace rapporto rischio/beneficio ed infine il potenziale ruolo degli antagonisti della vitamina $\mathrm{K}$ nella patogenesi delle calcificazioni vascolari, ${ }^{14}$ hanno portato ad un ridotto utilizzo degli anticoagulanti in tali soggetti. Per tale motivo, l'introduzione sul mercato dei nuovi anticoagulanti orali (l'inibitore diretto della trombina Dabigatran ed i 3 inibitori diretti del fattore Xa Apixaban, Rivaroxaban ed Edoxaban), meglio conosciuti come anticoagulanti orali diretti (direct oral anticoagulats, DOACs), ha destato interesse anche per il loro impiego nella popolazione dei pazienti affetti da malattia renale.

Le quattro molecole posseggono peculiari caratteristiche di carattere farmacologico come evidenziato nella figura 1.

Il profilo di sicurezza ed efficacia dei DOACs nei confronti del Warfarin è ormai ben stabilito anche nei pazienti con compromissione della funzione renale, e sono sempre più frequenti le segnalazioni di un loro impiego perfino in pazienti con CKD in stadio 4 (con eGFR compreso tra 15 e $29 \mathrm{~mL} / \mathrm{min}$ ), come suggerito anche dalle più recenti raccomandazioni della European Heart Rhytmn Association (EHRA) del 2018 (figura 2). ${ }^{15}$ 


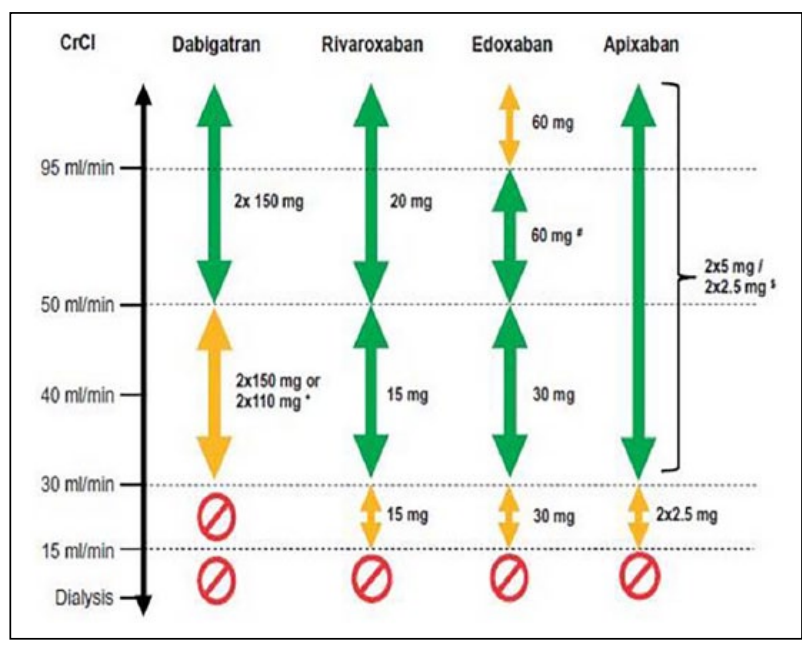

Figura 2. Raccomandazioni pratiche EHRA 2018.

\section{RIVAROXABAN: II DOAC ideale nel paziente con CKD?}

Nell'ambito dei DOACs, Rivaroxaban è una delle molecole più studiate nel paziente con malattia renale. Si tratta di un inibitore diretto del fattore Xa. Circa il 36\% del farmaco viene eliminato attraverso il rene; la quota restante viene convertita in composti inattivi ad opera del fegato. ${ }^{16}$ Nello studio randomizzato controllato ROCKET-AF, comprendente 14,264 pazienti con FA, rischio di stroke moderato-alto e $\mathrm{CrCl}>30 \mathrm{~mL} / \mathrm{min}$, Rivaroxaban $(20 \mathrm{mg} / \mathrm{die})$ si è dimostrato non inferiore a Warfarin nella prevenzione dello stroke e dell'embolismo sistemico senza alcuna significativa differenza tra i 2 gruppi relativamente alle complicanze emorragiche. Il gruppo di pazienti con CKD lieve-moderata ( $\mathrm{CrCl}$ compresa tra 30 e $39 \mathrm{~mL} / \mathrm{min}$ ) a cui vennero somministrati $15 \mathrm{mg} /$ die di Rivaroxaban non mostrò differenze, in termini di outcome, rispetto alla coorte con normale funzione renale trattata con $20 \mathrm{mg} / \mathrm{die}$ di Rivaroxaban. ${ }^{17}$ Inoltre, sempre nello stesso gruppo, il dosaggio di $15 \mathrm{mg}$ ha permesso di evidenziare un profilo farmacocinetico e farmacodinamico perfettamente sovrapponibile a quello del dosaggio di $20 \mathrm{mg} / \mathrm{die}$ nei soggetti con funzione renale conservata. ${ }^{17}$ Il rischio di sanguinamenti maggiori era praticamente sovrapponibile nella popolazione con CKD trattata con Warfarin o Rivaroxaban. ${ }^{17}$ Rivaroxaban è stato approvato dalla Food and Drug Administration (FDA) nel novembre 2011 per l'utilizzo in pazienti con funzione renale normale ed in quelli affetti da CKD con $\mathrm{CrCl}$ compresa fra $15 \mathrm{e}$ $50 \mathrm{~mL} / \mathrm{min}$ al dosaggio di $20 \mathrm{mg}$ e $15 \mathrm{mg}$ una volta al giorno, rispettivamente. Un aumento di circa il 52\% della concentrazione plasmatica, e del $26 \%$ del picco di concentrazione, furono riscontrati in 8 pazienti con CKD (eGFR medio di $43 \mathrm{~mL} / \mathrm{min} / \mathrm{m}^{2}$ ) dopo una singola somministrazione di $10 \mathrm{mg}$ di Rivaroxaban. ${ }^{1}$ Tuttavia, non si è avuto nessun aumento del rischio emorragico nei pazienti con CKD moderata trattati con $15 \mathrm{mg}$ al giorno di Rivaroxaban rispetto al Warfarin. ${ }^{16}$

Attualmente Rivaroxaban non viene raccomandato, in accordo alle linee guida europee, per i pazienti con ESRD $(\mathrm{CrCl}<15 \mathrm{~mL} / \mathrm{min})$ o sottoposti a trattamento dialitico cronico, in quanto solo una piccola parte del farmaco viene rimosso con i trattamenti ad alti flussi. ${ }^{18}$

La FDA ha invece approvato nel 2016 l'impiego di Rivaroxaban anche nel paziente con eGFR $<15 \mathrm{~mL} / \mathrm{min}$ e nel paziente sottoposto a trattamento sostitutivo della funzione renale. ${ }^{19}$

Le linee guida dell'American Heart Association (AHA) del 2014 suggeriscono come, nei pazienti con eGFR $<15$ / $\min / 1.73 \mathrm{~m}^{2}$, il farmaco da prendere in considerazione (classe di raccomandazione IIIB) sia il Warfarin, soprattutto in quelli che presentano uno score $\mathrm{CHA}_{2} \mathrm{DS}_{2}-\mathrm{VASc} \geqslant 2$. Si tratta, però, di un suggerimento non universalmente condiviso, tant'è vero che la Canadian Cardiovascular Society, aggiornando le proprie linee guida, ha raccomandato di non applicare in modo routinario i protocolli di anticoagulazione nei pazienti affetti da fibrillazione atriale non valvolare con valore di filtrato glomerulare $<30 \mathrm{~mL} / \mathrm{min} / 1.73 \mathrm{~m}^{2} .{ }^{20}$ Considerate le premesse, è arrivato probabilmente il momento di cominciare a considerare delle strategie alternative anche per una popolazione di pazienti ad alto rischio come quelli affetti da ESRD e sottoposti a trattamento emodialitico, anche alla luce delle sopraindicate indicazioni della FDA.

Un primo studio, ad opera di Dias e colleghi, ha permesso di analizzare, dal punto di vista farmacodinamico e farmacocinetico, l'impiego di Rivaroxaban in pazienti emodializzati, concludendo che le curve dose-risposta e quelle di eliminazione non si differenziano in maniera statisticamente significativa rispetto a quelle osservate nella popolazione con eGFR $>30 \mathrm{~mL} / \mathrm{min} / 1.73 \mathrm{~m}^{2} .{ }^{21}$

Rivaroxaban possiede una doppia via di eliminazione (67\% epatica e $33 \%$ renale) e il $66 \%$ dell'intera molecola viene processato a livello epatico tramite il citocromo P450; i relativi metaboliti vengono, a loro volta, eliminati sia per via fecale, sia per via urinaria in una percentuale paritaria del $50 \%$. Circa $1 / 3$ della molecola (il $33 \%$ come già detto) rappresenta la porzione immodificata eliminata per via renale grazie a specifici trasportatori. ${ }^{16}$ Il peso molecolare di 435 Da ed uno scarso volume di distribuzione (pari a circa 50 L) rappresentano caratteristiche di Rivaroxaban in grado di ritenerlo potenzialmente dializzabile; a far da contraltare, però, contribuisce l'elevato legame proteico $(>90 \%)$ il quale ne impedisce la rimozione anche con i dializzatori ad alto flusso.

Le modificazioni evidenziate nelle curve di farmacocinetica e farmacodinamica si sono mostrate sovrapponibili a quelle evidenziate in studi precedenti effettuati su pazienti con compromissione moderata e/o severa della funzione renale, a testimonianza del fatto che l'impatto del trattamento emodialitico potrebbe non essere 


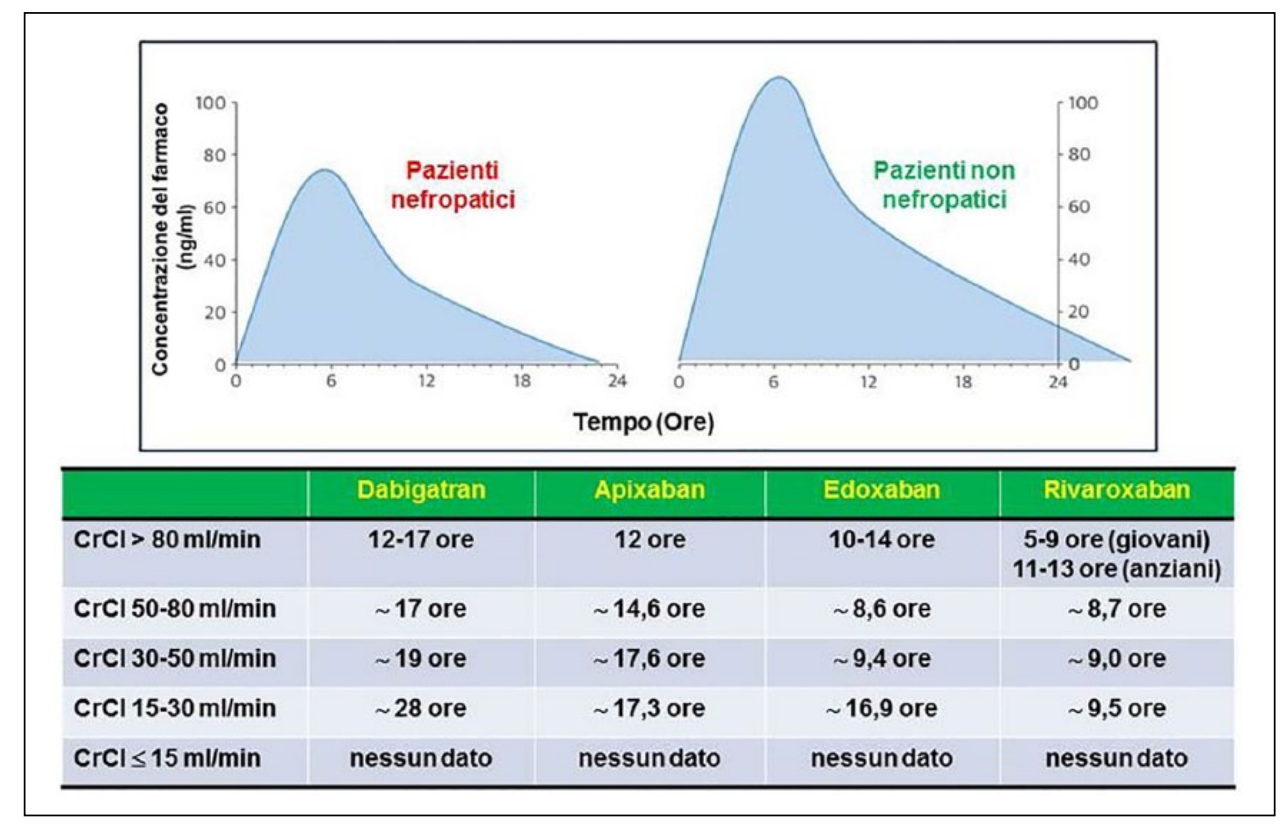

Figura 3. Emivita plasmatica dei singoli DOACs.

così negativo. ${ }^{21}$ A conferma di ciò, la somministrazione di Rivaroxaban prima o dopo la dialisi non impatta sulle curve di farmacodinamica. ${ }^{21}$ Inoltre, lo studio di De Vriese ha evidenziato un altro elemento che va tenuto in debita considerazione nella prescrizione di Rivaroxaban in pazienti con compromissione della funzione renale: man mano che diminuisce il valore del filtrato glomerulare, l'emivita plasmatica del farmaco non si modifica, attestandosi (fino a valori di eGFR $>15 \mathrm{~mL} / \mathrm{min} / 1.73 \mathrm{~m}^{2}$ ) costantemente sulle 9 ore, al contrario di quanto avviene con gli altri DOACs. ${ }^{18}$

Nel corso del 2019 sarà disponibile anche per Rivaroxaban, così come per gli altri inibitori del fattore $\mathrm{Xa}$, un antidoto specifico per l'eventuale sovraesposizione al farmaco medesimo: si tratta di Andexanet che è risultato in grado di ridurre del 92\% l'attività anti-fattore Xa di Rivaroxaban. $^{22}$ Di recentissima pubblicazione sul New England Journal of Medicine i risultati di un trial clinico condotti su oltre 300 pazienti che hanno evidenziato il ruolo di Andexanet come antidoto di provata efficacia in caso di sovradosaggio di Rivaroxaban. ${ }^{23}$

Per quanto concerne le prospettive future, nel mese di Aprile 2016 è iniziato il trial Xareno (Factor XA-inhibition in RENal patients with non-valvular atrial fibrillation Observational registry) che ha arruolato 2,500 pazienti allo scopo di valutare il profilo di efficacia e sicurezza di Rivaroxaban in una coorte di pazienti affetti da malattia renale cronica con valori di eGFR compresi tra 15 e $49 \mathrm{~mL} / \mathrm{min} / 1.73 \mathrm{~m}^{2}$ (ClinicalTrails.gov identifier: NCT02663076). Si tratta, per l'appunto, di uno studio di coorte, prospettico con un periodo di follow-up di 18 mesi che ha come end-point primari il declino del filtrato glomerulare, episodi di sanguinamenti maggiori, mortalità per tutte le cause, eventi cerebrovascolari (TIA, stroke), eventi cardiovascolari maggiori (MACE) ed episodi di tromboembolismo venoso profondo.

Rivaroxaban è l'unica molecola, appartenente alla classe dei DOACs, che possiede dati riguardanti ogni tipologia di studio clinico a partire dai database retrospettivi (ad es. REVISIT-US) fino ai trials clinici randomizzati e controllati (ROCKET-AF) (figura 2). ${ }^{24}$

Tra i NOACs, Rivaroxaban si caratterizza per un programma di studi particolarmente articolato in cui la mole di dati relativa a oltre 275,000 pazienti derivati da studi randomizzati e da analisi "real world" condotti in contesti diversi, testimonia la robustezza delle evidenze in merito a sicurezza ed efficacia di questo farmaco anticoagulante orale. I dati di "real world" documentano inoltre livelli di persistenza e aderenza al trattamento con Rivaroxaban addirittura migliori di quelli degli studi randomizzati controllati.

Dal punto di vista strettamente nefrologico, la sicurezza della molecola è documentata, oltre che dagli studi di Dias ${ }^{21}$, anche da quanto pubblicato da Heidbuchel et al (figura 3). ${ }^{25}$ Questi ultimi autori hanno chiaramente evidenziato come, rispetto alle altre molecole delle stessa classe, il profilo farmacocinetico e farmacodinamico di Rivaroxaban si mantenga costante con un'emivita sostanzialmente immodificata nel passaggio da una condizione di normofunzione renale fino ad uno stadio 4 di CKD (valori di eGFR compresi tra 15 e $30 \mathrm{~mL} / \mathrm{min}$ ) (figura 4). ${ }^{25}$

Tra le altre osservazioni, la recente approvazione all'impiego di Rivaroxaban nei pazienti con ESRD da parte della FDA apre ulteriori scenari per l'impiego del 


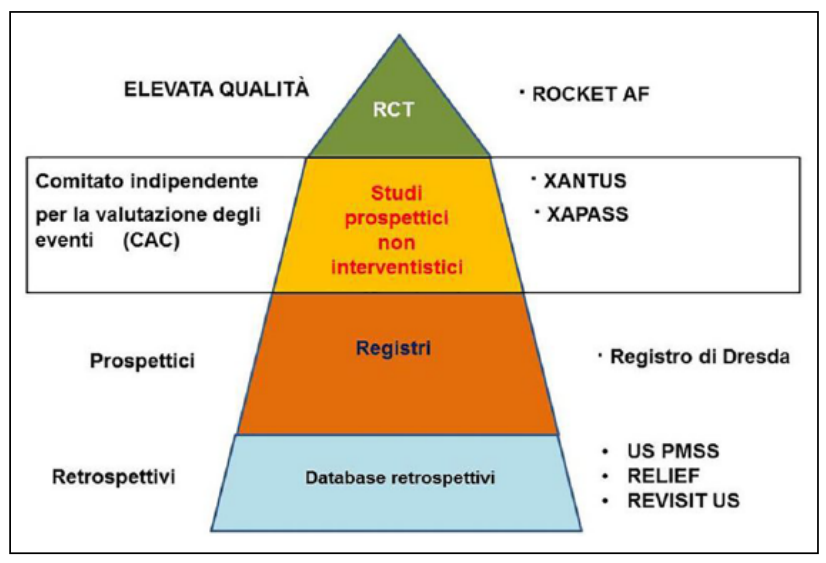

Figura 4. Piramide degli studi clinici condotti con Rivaroxaban.

suddetto NOAC in una popolazione di pazienti estremamente "complicata". ${ }^{26}$

Un recente studio di Yao e colleghi, pubblicato nel mese di Febbraio 2018, ha inoltre evidenziato come, in uno studio di popolazione, Rivaroxaban presenti la minore incidenza di eventi renali acuti con un impatto inferiore, rispetto agli altri NOACs, in termini di declino del filtrato glomerulare: pur con tutte le limitazioni di uno studio di tale natura, si tratta di un elemento di cui tener conto. ${ }^{27}$

Sempre la stessa pubblicazione ha, inoltre, evidenziato come Rivaroxaban presenti degli ottimi outcome anche per quanto concerne il raddoppio dei livelli di creatininemia rispetto a quelli basali pre-trattamento. ${ }^{27}$

Un altro studio recente, pubblicato da Loo e colleghi su $\mathrm{BMJ}$, ha confermato i dati positivi riguardanti l'impiego di Rivaroxaban nei pazienti affetti da CKD; anche in questo studio di popolazione, la molecola ha evidenziato un buon profilo di efficacia e sicurezza con una percentuale di sanguinamenti maggiori e di ictus emorragici paragonabili a quelli documentati nei pazienti in terapia con Warfarin. ${ }^{12}$

Interessante anche la recente pubblicazione di Huang e colleghi inerente a uno studio di "real world" condotto su oltre 24,000 pazienti asiatici (dei quali oltre $20 \%$ circa presentava diversi gradi di compromissione renale) allo scopo di valutare l'efficacia e la sicurezza di tre diversi dosaggi di Rivaroxaban. I risultati hanno evidenziato come i soli dosaggi di 20 e $15 \mathrm{mg}$ in mono-somministrazione giornaliera fossero associati ad un rischio minore di emorragia intracranica e di episodi tromboembolici (con evidenze comparabili anche nei pazienti con compromissione della funzione renale), al contrario del dosaggio di $10 \mathrm{mg} .{ }^{28}$

Un altro punto a favore di Rivaroxaban risiede, soprattutto in una popolazione di pazienti in politrattamento farmacologico quali i pazienti affetti da CKD, nella monosomministrazione giornaliera, garanzia di migliore compliance e, quindi, di outcome clinici più favorevoli. ${ }^{29}$
Infine, un recente position paper congiunto NKFKDOQI ha affermato come, nel paziente con ESRD, anche Rivaroxaban, come Apixaban, possa essere utilizzato (al dosaggio di $15 \mathrm{mg} / \mathrm{die}$ ) in caso di fibrillazione atriale non valvolare.

Indubbiamente interessanti $\mathrm{i}$ dati di uno studio osservazionale, retrospettivo e longitudinale, condotto su due coorti di pazienti con CKD in stadio $3 \mathrm{~A} / 4$ nei quali sono stati messi a confronti gli end-point di sicurezza ed efficacia di Rivaroxaban $(n=247)$ vs Warfarin $(n=101)$ : dai risultati preliminari emerge in maniera inequivocabile la superiorità di Rivaroxaban per quanto riguarda entrambi gli end-point prefissati di sicurezza ed efficacia. Entrando più nei particolari è emerso come nel gruppo Warfarin si siano osservati ben 25 episodi ischemici cerebrali ( 15 su base emorragica e 10 su base ischemica) a fronte dell'assenza totale di tali eventi nel gruppo trattato con Rivaroxaban. Sempre nel gruppo trattato con Warfarin si è assistito anche a 5 episodi di trombosi venosa profonda (nessuno nel gruppo trattato con Rivaroxaban) con 8 casi di emorragia gastrointestinale (contro i 2 del gruppo Rivaroxaban). ${ }^{30}$

Rivaroxaban, inoltre, ha evidenziato anche degli effetti pleiotropici in quanto è stato dimostrato in maniera piuttosto inequivocabile come i pazienti trattati con il suddetto inibitore del fattore Xa, rispetto ai soggetti trattati con Warfarin, presentino una maggiore incidenza di calcificazioni valvolari cardiache. I risultati evidenziano chiaramente come si assista ad un rallentamento della progressione (ma anche ad una regressione) delle calcificazioni mitraliche ed aortiche in rapporto direttamente proporzionale con la riduzione dei livelli sierici di proteina C- reattiva. Quest'ultimo aspetto si spiega piuttosto facilmente con il fatto che l'inibizione del fattore Xa (potente induttore dello stato infiammatorio) determina una riduzione del grado di infiammazione cronica, proprio il "pabulum" sul quale si impianta, nel paziente affetto da CKD, la calcificazione vascolare/ cardiaca. $^{31}$

\section{Conclusioni}

Anche nel paziente con CKD la terapia anticoagulante con DOACs ha aperto nuove strade per la gestione della profilassi delle complicanze tromboemboliche legate alla fibrillazione atriale. ${ }^{32}$ Tutte e 4 le molecole, pur presentando delle caratteristiche farmacologiche diverse, si sono dimostrate efficaci e sicure nel paziente con CKD. Rivaroxaban sembra possedere, al contempo, alcune peculiarità (dosaggio specifico, mono-somministrazione, effetti pleiotropici) che lo rendono particolarmente "appetibile" per l'impiego in una così particolare popolazione di pazienti.

\section{Dichiarazione di assenza di conflitto di interessi}

Gli Autori dichiarano di non avere conflitti di interessi. 


\section{Finanziamenti}

Gli Autori dichiarano di non aver ricevuto finanziamenti specifici da qualsiasi ente nei settori pubblico, privato o senza fini di lucro.

\section{Bibliografia}

1. Kannel WB, Abbott RD, Savage DD and McNamara PM. Epidemiologic features of chronic atrial fibrillation: the Framingham study. N Engl J Med 1982; 306:1018-1022.

2. Wolf PA, Benjamin EJ, Belanger AJ, Kannel WB, Levy D, et al. Secular trends in the prevalence of atrial fibrillation: the Framingham study. Am Heart J 1996; 131:790-795.

3. Kannel WB, Wolf PA, Benjamin EJ and Levy D Prevalence, incidence, prognosis, and predisposing conditions for atrial fibrillation: population-based estimates. Am J Cardiol 1998; $82: 2 \mathrm{~N}-9 \mathrm{~N}$.

4. Salam AM, Albinali HA, Al-Sulaiti EM, et al. Effect of Age on Treatment, Trends and Outcome of Patients Hospitalized With Atrial Fibrillation: Insights From a 20-Years Registry in a Middle-eastern Country (1991-2010). Aging Clin Exp Res 2012; 24(6):682-90.

5. Salam AM, Albinali HA, Al-Mulla AW, et al. Women hospitalized with atrial fibrillation: Gender differences, trends and outcome from a 20-year registry in a middle eastern country (1991-2010). Int J Cardiol 2012; 168(2):975-80.

6. Salam AM, Albinali HA, Al-Mulla AW, Singh R and Al Suwaidi J. Secular Trends, Treatments, and Outcomes of Middle Eastern Arab and South Asian Patients Hospitalized With Atrial Fibrillation: Insights From a 20-Year Registry in Qatar (1991-2010). Angiology 2012; 64(7):498-504.

7. Go SA, Chertow MG, Fan D, McCulloch CE and Hsu CY. Chronic kidney disease and the risks of death, cardiovascular events, and hospitalization. N Engl J Med 2004; 351:12961305.

8. Sarnak MJ, Levey AS, Schoolwerth AC, et al. Kidney disease as a risk factor for development of cardiovascular disease: a statement from the American Heart Association Councils on Kidney in Cardiovascular Disease, High Blood Pressure Research, Clinical Cardiology, and Epidemiology and Prevention. Hypertension 2003; 42:1050-1065.

9. Vazquez E, Sanchez-Perales C, Garcia-Garcia F, et al. Atrial fibrillation in incident dialysis patients. Kidney Int 2009; 76:324-330.

10. Go AS, Chertow GM, Fan D, et al. Chronic kidney disease and the risks of death, cardiovascular events and hospitalization. N Engl J Med 2004; 351:1296-1305.

11. Nakayama M, Metoki $H$, Terawaki $H$, et al. Kidney dysfunction as a risk factor for first symptomatic stroke events in a general Japanese population - the Ohasama study. Nephrol Dial Transplant 2007; 22:1910-1915.

12. US renal Data System: USRDS 2006 Annual data report: Atlas of End - Stage Renal Disease in the United States, Bethesda National Institute of Health, National Institute of Diabetes and Digestive and Kidney Disease, 2006.

13. Vazquez E, Sanchez-Perales C, Lozano C, et al. Comparison of prognostic value of atrial fibrillation versus sinus rhythm in patients on long-term hemodialysis. Am J Cardiol 2003; 92: 868-871.
14. De Vriese AS, Caluwè R and Raggi $P$. The atrial fibrillation conundrum in dialysis patients. Am Heart $J$ 2016; 174: 111-9.

15. Steffel J, Verhamme P, Potpara TS, et al. ESC Scientific Document Group. The 2018 European Heart Rhythm Association Practical Guide on the use of non-vitamin $\mathrm{K}$ antagonist oral anticoagulants in patients with atrial fibrillation. Eur Heart J 2018; 39(16):1330-1393.

16. Kubitza D, Becka M, Mueck W, et al. Effects of renal impairment on the pharmacokinetics, pharmacodynamics and safety of rivaroxaban, an oral, direct factor Xa inhibitor. Br J Clin Pharmacol 2010; 70:703-12.

17. Fox KAA, Piccini JP, Wojtyla D, et al. Prevention of stroke and systemic embolism with rivaroxaban compared with warfarin in patients with non-alular atrial fibrillation and moderate renal impairment. Eur Heart J 2011; 32:2387-94.

18. De Vriese AS, Caluwé R, Bailleul E, et al. Dose-finding study of rivaroxaban in hemodialysis patients. Am J Kidney Dis 2015; 66:91-8.

19. Hu A, Niu J and Winkelmayer WC. Oral Anticoagulation in Patients With End-Stage Kidney Disease on Dialysis and Atrial Fibrillation. Semin Nephrol 2018; 38(6):618-628.

20. Verma A, Cairns JA, Mitchell LB, et al. 2014 focused update of the Canadian cardiovascular society guidelines for the management of atrial fibrillation. Can J Cardiol 2014; 30:1114-1130.

21. Dias C, Moore KT, Murphy J, et al. Pharmacokinetics, pharmacodynamics, and safety of single-dose rivaroxaban in chronic hemodialysis. Am J Nephrol 2016; 43(4):229-36.

22. Siegal DM, Curnutte JT, Connolly SJ, et al. Andexanet alfa for the reversal of factor Xa inhibitor activity. $N$ Engl J Med 2015; 373:2413-2424.

23. Connolly SJ, Crowther M, Eikelboom JW and Gibson CM for the ANNEXA-4 Investigators. Full Study Report of Andexanet Alfa for Bleeding Associated with Factor Xa Inhibitors. $N$ Eng $J$ Med February 2019 doi: 10.156/ NEJMoa1814051

24. Colivicchi F. Dagli studi clinici alla pratica clinica: come le evidenze di "real world" completano il profile di efficacia e sicurezza di rivaroxaban. G Ital Cardiol 2017; 18(2 Suppl. 2): $3 \mathrm{~S}-11 \mathrm{~S}$.

25. Heidbuchel H, Verhamme P, Alings $M$, et al. Updated European Heart Rhythm Association Practical Guide on the use of non-vitamin $\mathrm{K}$ antagonist anticoagulants in patients with non-valvular atrial fibrillation. Europace 2015; 17(10):1467-507.

26. Klil-Drori AJ and Tagalakis V. Direct Oral Anticoagulants in End-Stage Renal Disease. Semin Thromb Hemost 2018; 44(4):353-363.

27. Yao X, Tangri N, Gersh BJ, Sangaralingham LR, Shah $\mathrm{ND}$, Nath KA and Noseworthy PA. Renal Outcomes in Anticoagulated Patients With Atrial Fibrillation. J Am Coll Cardiol 2017; 70(21):2621-2632.

28. Huang Y, Mai L, Zhang Y and Cai X. Optimal Dose of Rivaroxaban in Asians With Atrial Fibrillation: Further Studies Are Warranted. J Am Coll Cardiol 2018; 72(21): 2681-2682.

29. Vimalesvaran K, Dockrill SJ and Gorog DA. Role of rivaroxaban in the management of atrial fibrillation: insights 
from clinical practice. Vasc Health Risk Manag 2018; 14:13-21.

30. Di Lullo L, Tripepi G, Ronco C, et al. Safety and effectiveness of rivaroxaban and warfarin in moderate-to-advanced CKD: real world data. J Nephrol 2018; 31(5):751-756.

31. Di Lullo L, Tripepi G, Ronco C, et al. Cardiac valve calcification and use of anticoagulants: Preliminary observation of a potentially modifiable risk factor. Int $J$ Cardiol 2019; 278:243-249.

32. Di Lullo L, Floccari F, Ravera R, et al. Gli anticoagulanti orali non vitamina K-dipendenti nei pazienti con fibrillazione atriale non valvolare ed end-stage renale disease (ESRD): un matrimonio impossibile? G Tec Nefrol Dial 2017; 29(2):117-26. 